\title{
Propuesta de Implementación de un Modelo de Teletrabajo
}

\author{
Alejandro Villafrade Vargas ${ }^{1}$, Jose Ignacio Palacios Osma ${ }^{1}$ \\ alejandro_villafrade@hotmail.com,jpalacios@udistrital.edu.co \\ ${ }^{1}$ Grupo de Investigación en Comercio Electrónico en Colombia - GICOECOL, Universidad Francisco Jose de \\ Caldas, Bogota, Colombia.
}

DOI: 10.4304/risti.12.17-31

\begin{abstract}
Resumen: El Teletrabajo hoy en día es una realidad, que tiene muchos beneficios relacionados con la eficiencia, la productividad, la sostenibilidad y la satisfacción laboral entre otros, sin embargo no es una forma de trabajo generalizada y estructurada en las organizaciones debido a diversas percepciones al respecto. Esta modalidad de trabajo plantea cambios y barreras a las que se enfrentan las organizaciones y los empleados al momento de apropiar e incorporar el teletrabajo, el presente estudio presenta los principales obstáculos o amenazas que implica la incorporación del teletrabajo en la organización.
\end{abstract}

Palabras-clave: Teletrabajo, Tecnologías de la Información.

\section{Proposal for Implementing a Telecommuting Model}

Abstract: Currently Teleworking is a reality that has many benefits related to efficiency, productivity, sustainability and job satisfaction among others; however isn't a generalized form of structured job organizations because of different perceptions about it. This mode of job changes raises barriers to those faced by organizations and employees at the time for adopting and incorporate telework, this study shows the major obstacles or threats about the incorporation of telework in the organization.

Keywords: Telework, Information technology.

\section{Introducción}

Son innumerables los beneficios que presenta el teletrabajo como modalidad laboral para las organizaciones y las grandes ciudades capitales, pues a partir del desarrollo de las tecnologías de la información y las Comunicaciones (TIC), es posible establecer diferentes formas de organización y gestión empresarial, que llevan entre otros aspectos, a la reducción de costos de transacción, optimización de recursos, intercambio de información y conocimiento, y sobre todo un mayor reconocimiento a 
las capacidades y fortalezas del hombre como ser creador y autónomo. De allí que las empresas requieren de estrategias que mejoren la calidad de vida de los trabajadores, la productividad de las empresas y el desarrollo social de las ciudades, para propiciar la creación de un valor compartido (Porter \& Kramer, 2011).

El presente artículo presenta un modelo para implementar esta forma de trabajo, a fin de que las PYMES puedan aplicarla en sus organizaciones, teniendo en cuenta las condiciones laborales y la capacidad de la empresa para asumir cambios estratégicos en su estructura organizacional.

La historia del concepto de teletrabajo se remonta a los años 70, empieza con la búsqueda en la optimización de los recursos naturales no renovables, generando una reflexión en torno al consumo de combustible (Berkovic, Kosovac, \& Dzebo, 2013), en tal sentido se propone esta modalidad como la forma de llevar el trabajo al trabajador, en vez del trabajador al trabajo, encontrado grandes beneficios del teletrabajo no solo en el ámbito ambiental sino en la productividad laboral, la flexibilidad, el bienestar laboral y la reducción de costos, así como el posicionamiento de las empresas (Vari, Tagliavini, \& Ter-Oganesova, 2011), sin embargo su adopción requiere de un cuidadoso análisis por parte de la organización que desee así implementarlo (Illegems, Verbeke, \& S'Jegers, 2001).

\section{Conceptualización de Teletrabajo}

El teletrabajo significa más que una simple modalidad laboral, es toda una cultura del trabajo y sobre el trabajador (Neirotti, Paolucci, \& Raguseo, 2011). Una cultura que choca con la cultura presencial que todavía impera en el mundo de las organizaciones y en la mayoría de las empresas, pues para nuestra sociedad del conocimiento es un reto el cambio de perspectiva en donde la valoración del trabajo, la medición del rendimiento y las consideraciones sociales, operan siempre en la presencialidad (Pérez Sánchez \& Gálvez Mozo, 2009).

Las estrategias claves que necesitan las empresas para tener éxito, se basan en la organización del trabajo: distintos investigadores argumentan que en este entorno, las empresas tienen que ser más flexibles para competir con éxito en un medio donde hay que responder a las presiones externas; adaptándose a las fluctuaciones de la demanda, aumentando los niveles de productividad, adoptando nuevas tecnologías, vinculando más estrechamente la remuneración salarial con los resultados de la empresa y mejorando la calidad de los productos y servicios (Martínez, Pérez, de Luis Carnicer, \& Jiménez, 2006; Ruth \& Chaudhry, 2008).

Dicha flexibilidad laboral es la filosofía del teletrabajo, como práctica que brinda la facilidad a los empleados para organizar su trabajo, mediante el uso de las TIC, que posibilitan que los empleados y directivos puedan desempeñar su actividad laboral a distancia y en forma asincrónica. Por lo tanto, el teletrabajo lo posibilita la tecnología pero es fundamenta en la innovación organizativa y del sistema laboral, así como también en cambios en factores como psicológicos y éticos, e igualmente en los factores 
de los equipos de trabajo a descentralizados e interconectados (Bin, Zeying, Omland, $\mathrm{Na}$, \& Sun, 2009).

La evolución del teletrabajo ha sido acompañada de diferentes conceptualizaciones a través del tiempo y del lugar donde se desarrolla. La tabla 1 presenta una revisión de la bibliografía encontrada sobre el teletrabajo.

Tabla 1. Conceptualización de teletrabajo

\begin{tabular}{|l|l|}
\hline Autor & Definición \\
\hline (Nilles, 1988) & $\begin{array}{l}\text { "Cualquier forma de sustitución de desplazamientos relacionados con la } \\
\text { actividad laboral por tecnologías de la información" o, de forma más gráfica, } \\
\text { "la posibilidad de enviar el trabajo al trabajador, en lugar de enviar el } \\
\text { trabajador al trabajo" }\end{array}$ \\
\hline $\begin{array}{l}\text { La Organización } \\
\text { Internacional del } \\
\text { Trabajo (OIT) }\end{array}$ & $\begin{array}{l}\text { Forma de trabajo efectuada en un lugar alejado de la oficina central o del } \\
\text { centro de producción y que implica una nueva tecnología que permite la } \\
\text { separación y facilita la comunicación (Organización Internacional del } \\
\text { Trabajo, 2001). }\end{array}$ \\
\hline $\begin{array}{l}\text { Orero, Caamaño y } \\
\text { Arraibi }\end{array}$ & $\begin{array}{l}\text { Una forma flexible de organización del trabajo, que consiste en el } \\
\text { desempeño de la actividad profesional sin la presencia física del trabajador } \\
\text { en la empresa durante parte importante del horario laboral (Orero } \\
\text { Giménez, Caamaño Eraso, \& Arraibi Dañobeitia, 201o). }\end{array}$ \\
\hline $\begin{array}{l}\text { Hunton y Norman } \\
\text { (2010) }\end{array}$ & $\begin{array}{l}\text { El teletrabajo es un acuerdo de trabajo flexible que proporciona a los } \\
\text { empleados la capacidad para "realizar el trabajo para sus empleadores } \\
\text { periódica, regular o exclusivamente desde la casa o desde otra localización } \\
\text { remota que está equipada con la apropiada tecnología para transferir el } \\
\text { trabajo a la organización (Hunton \& Norman, 2010) }\end{array}$ \\
\hline Cónvenio sobre Sánchez & $\begin{array}{l}\text { El término teletrabajo, se consigna para aludir al trabajo en el que se } \\
\text { utilizan las TIC y que se lleva a cabo de manera regular fuera de las } \\
\text { instalaciones del empleador. Por tanto, el nuevo convenio cubre a } \\
\text { numerosos trabajadores "móviles" o itinerantes, aś como a los que } \\
\text { desarrollan su actividad en su domicilio" (CES, 20o2). }\end{array}$ \\
\hline Europea
\end{tabular}




\begin{tabular}{|l|l|}
\hline \multirow{2}{*}{ Lier Tom } & $\begin{array}{l}\text { El teletrabajo es una forma de trabajo donde los empleados pueden realizar } \\
\text { sus actividades de trabajo fuera de la sede de la empresa y en horarios } \\
\text { flexibles. Es una opción reconocida para las empresas que se enfrentan a } \\
\text { dificultades espaciales y crecimiento, lo que permite situaciones de trabajo } \\
\text { más flexibles y crear competitiva ventajas (Lier, De Witte, \& Macharis, } \\
\text { 2012). }\end{array}$ \\
\hline
\end{tabular}

Fuente: Elaboración propia

\section{Modalidades del Teletrabajo}

El teletrabajo no es una actividad que se desarrolle únicamente desde el hogar del teletrabajador, sino que tiene muchas variantes y posibilidades. En cuanto a sus modalidades, se clasifican básicamente en tres tipos: el trabajo en casa, el trabajo móvil y el trabajo en telecentros; cuyas descripciones se menciona a continuación (Castells M, 2005 ; Montelongo, Lobato, \& Yris, 2010).

- Teletrabajo en casa: Se tiene el domicilio particular como lugar de trabajo, estipulado en un contrato con el empleador y se refiere a la realización de tareas específicas desde el domicilio o despacho profesional.

- Trabajo móvil: También denominado como nómada o itinerante se refiere a aquellos trabajadores cuya actividad requiere frecuentes desplazamientos, desarrollan la mayor parte de su actividad en diferentes lugares.

- $\quad$ Trabajo en telecentros: También denominado como oficina remota, la cual es una oficina de recursos compartidos que dispone de las instalaciones de telecomunicaciones y de los equipos informáticos necesarios para desarrollar actividades de teletrabajo.

\section{Teletrabajo en Colombia}

El teletrabajo es una tendencia cada vez más común en nuestro país, tal como evidenció una encuesta realizada a más de 2.300 Colombianos en las principales ciudades del país (Universia Colombia, 2012). Dentro de las conclusiones más importantes, se destaca:

- $\quad \mathrm{Al} 71 \%$ les gustaría incursionar en la modalidad del teletrabajo, 21\% quisiera hacerlo pero sólo medio tiempo, 6\% quisiera trabajar desde la oficina y el $2 \%$ restante afirmó estar trabajando actualmente desde su casa.

- Ante lo positivo del teletrabajo, para el 55\% de los encuestados lo favorable del sistema es que permite lograr un equilibrio entre lo laboral y lo familiar. Para un $33 \%$, permite ahorrar tiempo y dinero y para un $10 \%$ este mecanismo permite evitar el estrés generado por la presión en la oficina. Tan sólo $2 \%$ de los colombianos no encuentra nada de positivo en el teletrabajo.

- Ante lo negativo, el $46 \%$ opinó que no tiene nada de malo, un $24 \%$ dijo que lo malo es que el día de mañana cuando quiera desempeñarse en una oficina no 
tendría background y por otro lado, el 20\% afirmó que el teletrabajo le impediría interactuar con otros profesionales.

Otra estudio realizado por la Corporación Colombia Digital (CCD, 2012), cuyo objetivo es implementar la línea base de Teletrabajo en empresas Colombianas es pertinente destacar algunos aspectos relevantes:

- Del total de las empresas que han implementado el teletrabajo, el $42 \%$ pertenece al sector servicios, el $30 \%$ al sector comercio, el $24 \%$ al sector industrial, el $3 \%$ al sector de la construcción y el $1 \%$ restante pertenece a otro sector diferente a los anteriores.

- De acuerdo al área de la empresa en las que se aplica el teletrabajo, el $51 \%$ corresponde al área comercial y ventas, el 23\% al área de producción, el 22\% al área administrativa y financiera, el $6 \%$ al área de mercadeo y el $17 \%$ restante corresponde a otra área diferente a las anteriores.

- La cultura organizacional que hace referencia principalmente al control que se ejerce sobre todo empleado en la modalidad laboral actual, es la razón por la que un $48 \%$ de quienes conocen el concepto de teletrabajo no ven viable su implementación.

- Según los encuestados las principales barreras en la implementación del teletrabajo son: inversión en tecnología e infraestructura en primer lugar, cultura organizacional en el segundo lugar, resistencia al cambio en tercer lugar y desconfianza en el cuarto lugar.

\section{Marco legal del teletrabajo en Colombia}

Colombia es de los pocos países a nivel mundial que ha generado propuestas de leyes sobre este tema. Por eso, en julio de 2008 el Congreso de la República de Colombia expidió la Ley 1221, que define el teletrabajo "como una forma de organización laboral, que consiste en el desempeño de actividades remuneradas o prestación de servicios a terceros mediante soportes TIC, para el contacto entre el trabajador y la empresa, sin requerirse la presencia física del trabajador en un sitio específico de trabajo" (Congreso de la Republica Colombia, 2008).

Dicha Ley se constituyó en el primer marco legal para el establecimiento de normas que promueven y regulan el teletrabajo, cuyo objeto consideró también la posibilidad de generación de empleo y autoempleo mediante la utilización de TIC, con lo cual se actualizan las formas o modalidades laborales en el país. Posteriormente en el año 2012 se emite el Decreto reglamentario de teletrabajo, el Decreto No 0884

De acuerdo a este marco jurídico y en consonancia con la reglamentación en la Unión Europea, siendo un modelo a seguir para todos aquellos países que carecen de legislación al respecto, se resaltan los siguientes puntos (Eraso, 2008):

- Los teletrabajadores son trabajadores de la empresa y por tanto deben recibir el mismo tratamiento que aquellos que trabajan dentro de las instalaciones de 
aquella y goza de las mismas condiciones legales y convencionales que los trabajadores de la empresa,

- El empleador tiene la obligación de realizar la adecuación del lugar destinado en el hogar para el teletrabajo, la instalación y mantenimiento de los equipos utilizados para el efecto, dándole sin embargo la libertad al trabajador de utilizar su propio equipo.

- El empleador regula el tema de la seguridad y la salud del teletrabajador disponiendo que el empleador es responsable de la salud y de la seguridad profesional del mismo.

\section{Metodología Investigación}

Iniciando el proceso de investigación el estudio adopto la investigación exploratoria, mediante la revisión documental buscando proporcionar conocimiento y entendimiento del teletrabajo en el ámbito mundial y nacional, que permitiera establecer el marco acerca del impacto en las Pymes Colombianas. La investigación incorpora diversos métodos para su análisis: se inició con un Panel de Expertos el cual se analizó mediante un análisis estructural. Posteriormente se estableció un diagrama de espina de pescado y análisis redes y conforme a los mismos se planteó un modelo de implementación.

\section{Resultados investigación}

El análisis estructural realizado a partir del panel de expertos, permito identificar las principales variables que afectan la incorporación del teletrabajo en la organización. El análisis estructural (Arcade, Godet, Meunier, Roubelat, \& Mendieta, 2004), realizado, comprendió tres etapas:

- Primera etapa: Consiste en enumerar el conjunto de variables que caracterizan el sistema estudiado y su entorno

- Segunda etapa: Reconstituir y describir la red de relaciones entre las variables/factores.

- Tercera etapa: Identificación de variables clave, esenciales a la evolución del sistema, en primer lugar mediante una clasificación directa, y posteriormente por una clasificación indirecta (llamada MIC-MAC para matrices de impactos cruzados Multiplicación Aplicada para una Clasificación).

Según la matriz de influencia-dependencia desarrollada, los factores que tienen mayor importancia en la creación del modelo de gestión para el teletrabajo son: cultura organizacional, inversión, desconfianza, seguridad de la información y normatividad respectivamente. Los resultados del panel de expertos se relacionan a continuación (Tabla 2): 
Tabla 2. Resultados panel de expertos

\begin{tabular}{|c|c|c|c|c|c|}
\hline & Desconfianza & Inversión & $\begin{array}{c}\text { Seguridad de la } \\
\text { información }\end{array}$ & Normatividad & $\begin{array}{c}\text { Cultura } \\
\text { organizacional }\end{array}$ \\
\hline Desconfianza & 0 & Influencia media & Influencia alta & Influencia media & Influencia media \\
\hline Inversión & Influencia media & 0 & Influencia alta & Influencia débil & Influencia alta \\
\hline $\begin{array}{c}\text { Seguridad de } \\
\text { la información }\end{array}$ & Influencia alta & Influencia alta & 0 & Influencia alta & Influencia media \\
\hline Normatividad & Influencia media & Influencia débil & Influencia débil & 0 & Influencia débil \\
\hline $\begin{array}{c}\text { Cultura } \\
\text { organizacional }\end{array}$ & Influencia alta & Influencia alta & Influencia alta & Influencia media & 0 \\
\hline
\end{tabular}

Fuente: Elaboración propia a partir del Panel de Expertos

Posteriormente se aplicó el programa MICMAC, método que tiene por objetivo, evidenciar las variables influyentes y dependientes a partir de la cual se identificó la relación entre las variables, tal como se observa en la tabla 3.

Tabla 3. Matriz de Influencia. Barreras del teletrabajo

\begin{tabular}{|c|c|c|c|c|c|}
\hline & 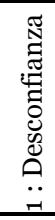 & 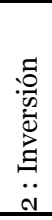 & 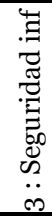 & 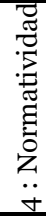 & 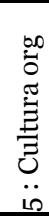 \\
\hline 1: Desconfianza & o & 2 & 3 & 2 & 2 \\
\hline 2 : Inversión & 2 & o & 3 & 1 & 3 \\
\hline 3 : Seguridad de la Información & 3 & 3 & o & 3 & 2 \\
\hline $4:$ Normatividad & 2 & 1 & 1 & o & 1 \\
\hline 5 : Cultura organizacional & 3 & 3 & 3 & 2 & o \\
\hline
\end{tabular}

Fuente: Elaboración Propia

Cada variable lleva asociado un indicador de motricidad (influencia) y un indicador de dependencia sobre todo el sistema. Estos valores se sitúan en un plano de motricidaddependencia (gráfico 1)

Como se observa en el grafico 1 la variable Cultura organizacional se encuentra ubicada en el sector 1, lo cual indica que es muy influyente y poco dependiente, es decir, es una variable explicativa que condiciona el sistema. Dentro del sector 2 se encuentran los factores Inversión, seguridad de la información y desconfianza, lo cual indica que son variables muy motrices (influyentes) y altamente dependientes, estableciendo un enlace inestable por naturaleza. En efecto, cualquier acción que se lleve a cabo sobre éstas, repercutirá sobre las demás y tendrá un efecto boomerang sobre ellas mismas que amplifica o desactiva el impulso inicial. 
La variable normatividad se encuentra ubicada en el sector 4, lo cual indica que es una variable poco influyente y poco dependiente. Aunque es una variable importante para la implementación de esquemas de teletrabajo.

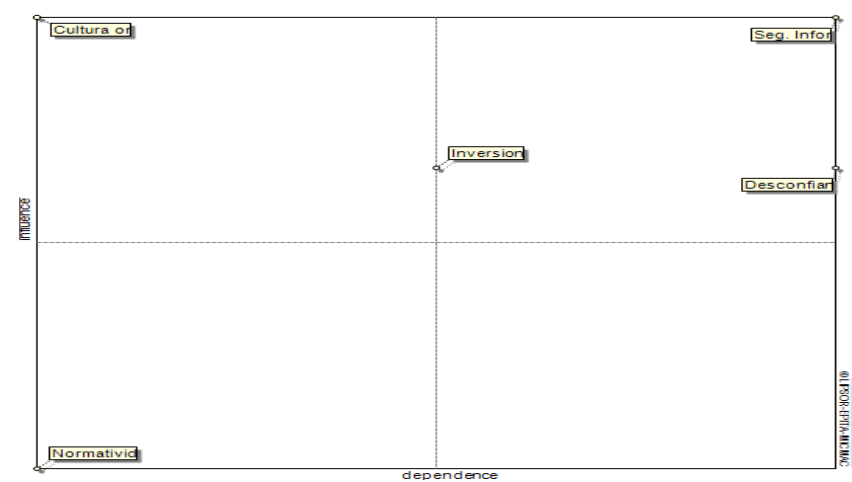

Grafico 1. Diagrama de Influencia directa/dependencia

A continuación se presenta el diagrama de redes o de correlación (Grafica 2) que explica la generación de las cuatro causas de primer nivel que contiene la espina de pescado.

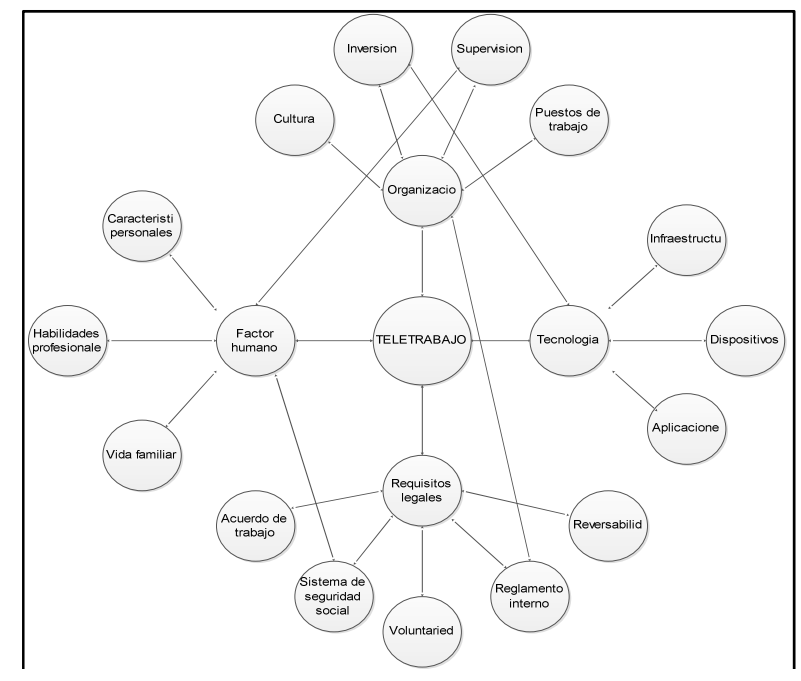

Grafico 2. Diagrama de correlación factores del teletrabajo

Teniendo en cuenta que en el diagrama de correlación; el nodo es un círculo que representa un aspecto importante de un problema y el arco es una línea que conecta dos nodos formando un esquema, estos cuatro factores fueron escogidos porque son los que presentan mayor número de relaciones, como se puede observar en la gráfica 2. 


\section{Análisis del Diagrama Causa - Efecto}

En primer lugar se tuvo en cuenta como eje principal de la espina de pescado el Teletrabajo, considerado como el tema principal de estudio y del cual se desprende la investigación que intenta explicar los factores que lo sustentan. Las causas de primer nivel que explican directamente el problema o efecto se clasificaron con el fin de tener un estudio global del problema (Grafico 3).

Las cuatro causas de primer nivel o principales, son:

- Requisitos legales: Esta causa tiene en cuenta ciertos requerimientos previos que se deben cumplir para implementar el modelo de teletrabajo de forma exitosa.

- Tecnología: Los requerimientos tecnológicos constituyen una herramienta necesaria para desarrollar del teletrabajo

- Factor humano: El factor humano es, tal vez el más importante, se debe contar con un conjunto de características personales y de competencias profesionales.

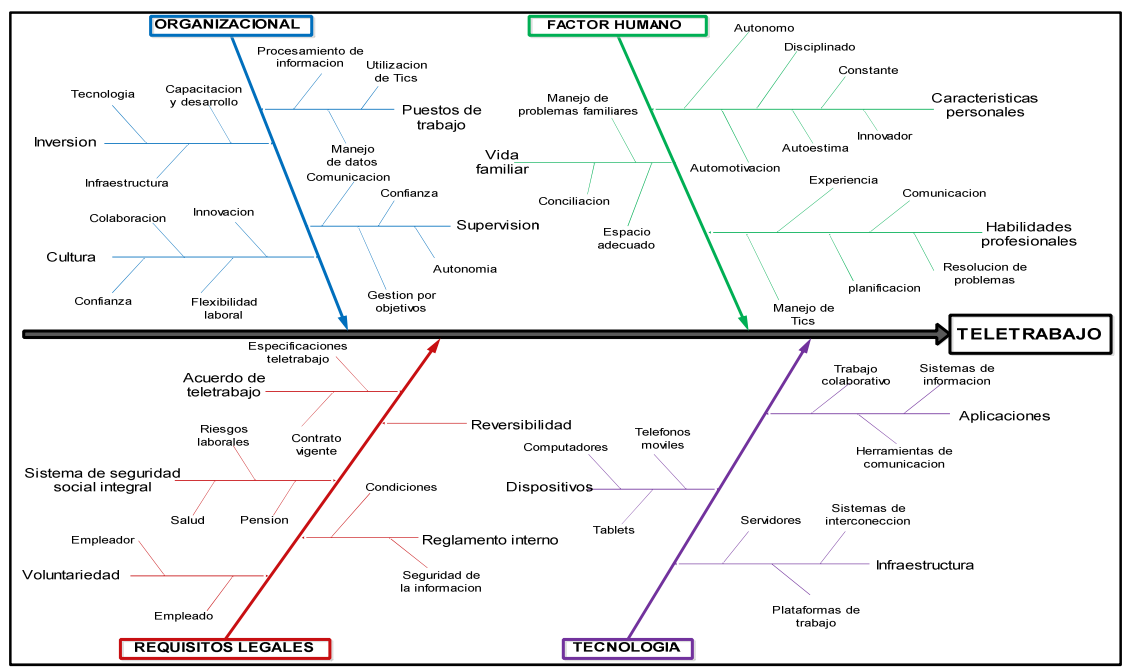

Grafico 3. Causa - Efecto

- Organizacional: Dentro de la organización hay una serie de aspectos de suma importancia como el sistema formal de tareas y relaciones jerárquicas que controla, coordina y motiva a los empleados para que cooperen para alcanzar las metas de una organización. 


\section{Modelo Propuesto}

El modelo de gestión para el teletrabajo define una política interna que fija objetivos con el fin de cambiar la cultura organizacional logrando incrementar la productividad laboral y mejorar la calidad de vida de los trabajadores.

La implementación de un sistema de teletrabajo no sólo consiste en la reubicación física de los empleados, requiere una serie de etapas, medidas y, sobre todo, de cambios culturales, además de los estrictamente técnicos, para lo cual se propone un modelo representado en el grafico 4.

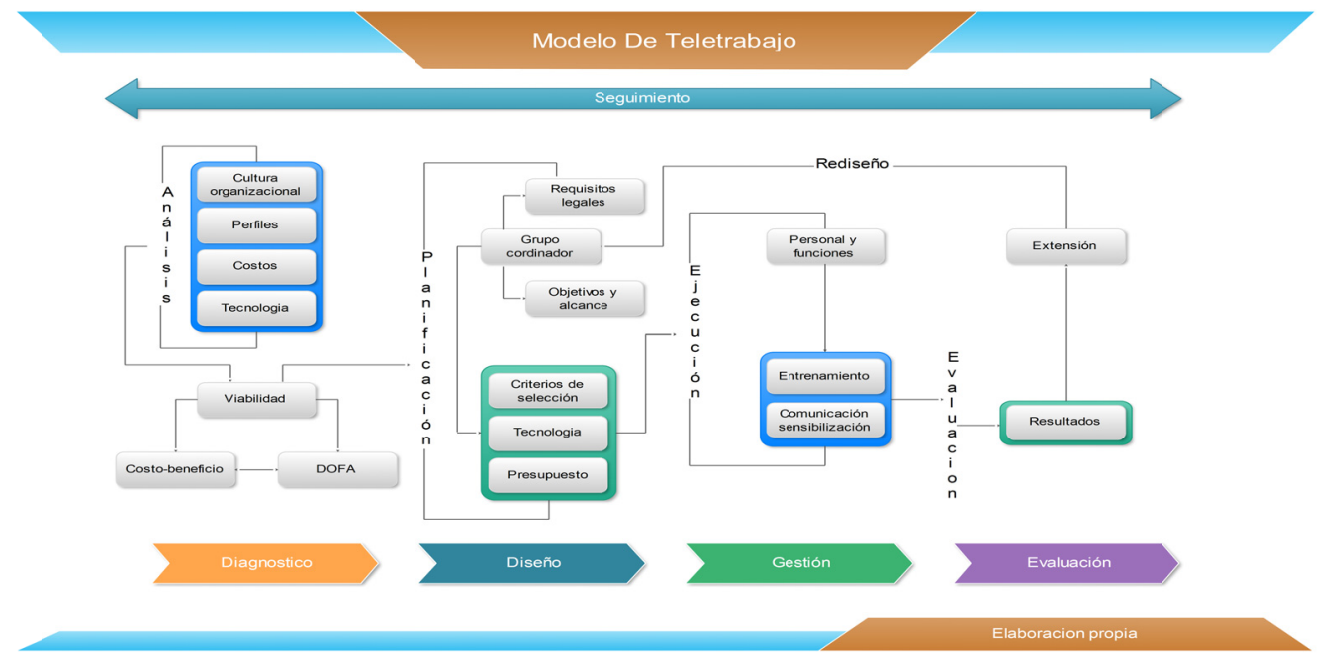

Grafico 4. Modelo de Gestión de Teletrabajo

\section{Fase I - Diagnostico}

El primer paso para la puesta en marcha de un programa de teletrabajo es conocer el estado actual de la organización y saber si se encuentra o no preparada para ello. En este sentido, se deben formular preguntas orientadas a conocer la percepción de los empleados y de la organización, en temas tales:

- Cultura organizacional: para esto deberá plantearse los siguientes interrogantes, ¿Considera que existe una relación entre la motivación de los empleados y su productividad?, entre otros

- Identificar Perfiles de: Supervisores, Cargos, Trabajadores

- Tecnología: Es importante validar si la organización cuenta con las herramientas tecnológicas necesarias.

- Costos: La organización deberá determinar si está dispuesta a adquirir nuevas herramientas tecnológicas e invertir. 
- Evaluación: Se deberá considerar el cumplimiento y existencia de cada uno de los puntos antes referidos y la medida del cómo influirán en la puesta en práctica del proyecto.

A continuación se presenta un análisis DOFA con el cual se pretende dar una visión genérica de las debilidades, amenazas, fortalezas y oportunidades que brinda la incorporación de esta nueva modalidad de trabajo (Grafico 5).

\begin{tabular}{|c|c|}
\hline FORTALEZAS & DEBILIDADES \\
\hline $\begin{array}{l}\text { - Flexibilidad en la organización de la empresa. } \\
\text { - Mayor confianza a los empleados y por ende mayor } \\
\text { - Mejora la prón. } \\
\text { - Reductividad de los empleados. } \\
\text { de la empresa. } \\
\text { - Innovación en la empresa. }\end{array}$ & $\begin{array}{l}\text { - Necesidad de la formación apropiada } \\
\text { - Telta de conocimiento sobre como dirigir el } \\
\text { - Pérdida de control de los empleados } \\
\text { - Eificultad de la comunicación entre los empleados } \\
\text { Escasa implementación de tecnología en la } \\
\text { emoresa }\end{array}$ \\
\hline OPORTUNIDADES & AMENAZAS \\
\hline $\begin{array}{l}\text { - Orear una nueva cultura organizacional. } \\
\text { - Obtención de ventajas competitivas por reducción } \\
\text { - Ge costes. } \\
\text { - } \quad \text { bajo costes posibilidades de expansión comercial a } \\
\text { - Reducción de costos de funcionamiento. }\end{array}$ & $\begin{array}{l}\text { - Riesgos laborales. } \\
\text { - } \text { mificultades del trabajador para adaptarse al } \\
\text { - Riesgos y por ende baja en la productividad. } \\
\text { la información por parte del trabajador. } \\
\text { - Pérdida del sentido de pertenencia del trabajador } \\
\text { respecto de la organización. }\end{array}$ \\
\hline
\end{tabular}

Grafico 5. Análisis DOFA Teletrabajo

Luego de analizar la situación actual de la organización frente a las expectativas que tienen sobre el teletrabajo, se lograra identificar si es apta o no para su implementación. Posteriormente se procederá a la elaboración del informe final del diagnóstico, el cual incluirá los resultados arrojados y una serie de conclusiones que orienten sobre la capacidad de la organización para implementar el teletrabajo.

\section{Fase II - Diseño}

La fase de diseño del programa de teletrabajo es posiblemente la etapa más importante para la implementación del mismo, ya que con su ejecución es posible tener una visión clara y una descripción precisa del modelo a ejecutar, sus características, condiciones y requisitos. La referencia para realizar el diseño es precisamente el diagnóstico realizado en la fase anterior.

- Análisis del marco legal: Para empezar de manera gradual y razonable la implantación de un programa de teletrabajo

- Creación del grupo coordinador: la conformación de un grupo coordinador que servirá de guía en las distintas etapas de su ejecución

- Objetivos del programa: Para que el programa tenga éxito es muy importante identificar cuáles son los beneficios que se esperan.

- Alcance del programa: En función del tamaño y de las necesidades concretas de la organización. 
- Naturaleza de las tareas a realizar: establecer las tareas que van a realizar los teletrabajador, permite un mejor ajuste de las necesidades tecnológicas que supone la implantación del teletrabajo.

- Nivel de seguridad: Las exigencias de seguridad impuestas por la empresa como la utilización de datos o programas almacenados y ubicados fuera de su ordenador.

- Acuerdo de Teletrabajo: Es el documento donde el empleador y el empleado se ponen de acuerdo para la implementación el teletrabajo, en el texto se especifican las condiciones individuales en las que se va a desarrollar esta modalidad.

- Política de Teletrabajo: Es uno de los elementos más importantes en la implementación del Teletrabajo en una empresa. Tiene como objetivo que la organización conozca cuál es el procedimiento de la aplicación de esta nueva práctica.

\section{Fase III - Gestión}

Teniendo como base el diseño del programa, la siguiente etapa es su implementación. Lo ideal es seguir cada uno de los pasos proyectados como guía de implementación que permita visualizar fácilmente los aspectos a tener en cuenta y los pasos a seguir a la hora de poner en marcha el esquema de teletrabajo propuesto:

- Comunicación, sensibilización y cultura organizacional: Para poder implementar esquemas de teletrabajo es indispensable que se realicen actividades en las cuales se le explique, las características del proyecto.

- Selección de los teletrabajadores: Una vez aprobados los criterios de selección del proyecto piloto, estos se podrán en marcha y se seleccionarán los trabajadores.

- Entrenamiento de los participantes: El teletrabajo implica cambios en la forma de trabajar, la comunicación con la empresa se establece por vía telemática, mayor orientación hacia los resultados.

- Ejecución del proyecto: Una vez cumplidos los pasos previos la organización y especialmente los teletrabajadores estarán en condiciones de iniciar el proyecto. El papel del grupo coordinador es estar atento a cada una de las nuevas necesidades e inquietudes que surjan durante el proceso.

\section{Fase IV - Evaluación y Sostenibilidad}

Al finalizar la etapa de implementación del programa piloto, se deberá llevar a cabo una evaluación, la cual es fundamental para el éxito del proyecto. Puede efectuarse mediante diversas técnicas de recolección de información como entrevistas, encuestas, cuestionarios, diarios, grupos de discusión y todo tipo de sondeos que arrojen datos sobre el proceso. La finalidad es averiguar si el programa está teniendo el éxito previsto, para ello, se debe analizar cuáles son los aspectos que están funcionando y 
cuáles no, centrándose principalmente en la aceptación o rechazo que se está produciendo entre teletrabajadores y supervisores.

- Evaluación: la evaluación cuantitativa del programa se debe realizar teniendo en cuenta factores como la productividad, la rentabilidad y la satisfacción de los empleados y la organización antes y después de adoptado el modelo de teletrabajo,

- Sostenibilidad: grupo coordinador del programa junto con los encargados de dirigir las actividades de los teletrabajadores debería ir recogiendo a lo largo de la aplicación las principales impresiones de los participantes, sus logros y dificultades.

- Extensión: una vez finalizado el proyecto piloto y analizados los resultados, el siguiente paso será la de decidir si implantar de forma definitiva el teletrabajo.

\section{Conclusiones}

Tras la revisión bibliográfica acerca de los factores que influyen en la implementación del teletrabajo en las organizaciones, se logró establecer que los principales factores son la cultura organizacional, la desconfianza, la seguridad de la información, la normatividad y la inversión, teniendo en cuenta las diferentes perspectivas de autores de distinta naturaleza.

A través del panel de expertos, se corroboraron los principales factores que influyen en la implementación de teletrabajo. Gracias al conocimiento del tema por parte de los invitados, se logró cuantificar su grado de incidencia, lo que permitió jerarquizar su importancia y determinar el enfoque de cada uno, basados en sus experiencias en la implementación de programas de teletrabajo en empresas Colombianas.

La implementación de un proyecto de teletrabajo exige un esfuerzo para la organización, que debe ser expuesto a los empleados y reconocido por ellos, ya que implica gestión de recursos humanos y tecnológicos, saber identificar los perfiles adecuados para teletrabajar, los supervisores adecuados para dirigir a los teletrabajadores y administrar de forma coherente las cargas de trabajo para lograr una mayor eficiencia en la gestión de las mismas.

Con la presente investigación se logró evidenciar que el Teletrabajo en las organizaciones cada vez se expande más, debido a la globalización e internacionalización de las empresas y las necesidades de la sociedad en temas de conciliación y flexibilidad laboral. El principal efecto del teletrabajo se manifiesta en la gran cantidad de beneficios que este aporta tanto a la sociedad, como a las empresas y los empleados. 


\section{Referencias Bibliográficas}

Arcade, J., Godet, M., Meunier, F., Roubelat, F., \& Mendieta, M. (2004). Análisis estructural con el método MICMAC, y estrategia de los actores con el método MACTOR. Buenos Aires: BCNA.

Berkovic, M., Kosovac, A., \& Dzebo, A. (2013, 20-24 May 2013). Analysis methodologies of assessment of impact teleworking on the decrease carbon emissions of physical mobility. Paper presented at the Information \& Communication Technology Electronics \& Microelectronics (MIPRO), 2013 36th International Convention on.

Bin, H., Zeying, L., Omland, H. O., Na, A., \& Sun, J. (2009, 17-19 June 2009). Management performance of telework teams based on experimental methods. Paper presented at the Control and Decision Conference, 2009. CCDC '09. Chinese.

Castells M. (2005 ). La Era de la Información. Economía Sociedad y Cultura (Vol. 1,2,3). España.

CCD, C. D. (2012). El Libro blanco del Teletrabajo en Colombia. In V.-. CCD (Ed.). Bogotá. Retrieved from www.colombiadigital.net.

CES, U. U. y. C. (2002). Acuerdo Marco Europeo sobre Teletrabajo, from http://www.ugt.es/teletrabajo/teletrabajo.htm

Congreso de la Republica Colombia. (2008). Ley 1221 - Teletrabajo. Bogotá.

Eraso, A. G. B. (2008). Teletrabajo en España: acuerdo marco y Administración Pública. RIO: Revista Internacional de Organizaciones(1), 129-148.

Hunton, J. E., \& Norman, C. S. (2010). The impact of alternative telework arrangements on organizational commitment: insights from a longitudinal field experiment. Journal of Information Systems, 24(1), 67-90.

Illegems, V., Verbeke, A., \& S'Jegers, R. (2001). The organizational context of teleworking implementation. Technological Forecasting and Social Change, 68(3), 275-291. doi: http://dx.doi.org/10.1016/So040-1625(00)00105-O

Lier, T. V., De Witte, A., \& Macharis, C. (2012). The Impact of Telework on Transport Externalities: The Case of Brussels Capital Region. Procedia - Social and Behavioral Sciences, 54(0), 240-250. doi: http://dx.doi.org/10.1016/j.sbspro.2012.09.743

Martínez, A. M., Pérez, M. P., de Luis Carnicer, M. P., \& Jiménez, M. J. V. (2006). Teletrabajo y flexibilidad: efecto moderador sobre los resultados de la empresa. Cuadernos de Economía y Dirección de la Empresa(29), 229-262.

Montelongo, M. D. G., Lobato, B. M., \& Yris, C. A. B. (2010). Teletrabajo:¿ Hacia una nueva forma organizacional? Análisis Organizacional, 1. 
Neirotti, P., Paolucci, E., \& Raguseo, E. (2011, 20-21 June 2011). Diffusion of Telework: Myth or Reality? Some Stylized Facts on Telework Diffusion in Italian Firms. Paper presented at the Mobile Business (ICMB), 2011 Tenth International Conference on.

Nilles, J. M. (1988). Traffic reduction by telecommuting: A status review and selected bibliography. Transportation Research Part A: General, 22(4), 301-317.

Orero Giménez, A., Caamaño Eraso, J., \& Arraibi Dañobeitia, J. R. (2010). Impacto organizativo derivado de la implantación del teletrabajo en una organización. Dirección y Organización(24).

Organización Internacional del Trabajo. (2001). Tecnologia de la Información: Salvar la brecha digital. Revista Trabajo - OIT, 38, 36.

Pérez Sánchez, C., \& Gálvez Mozo, A. M. (2009). Teletrabajo y vida cotidiana: Ventajas y dificultades para la conciliación de la vida laboral, personal y familiar. Athenea Digital-Revista de pensamiento e investigación social(15), 57-79.

Pérez, S. C. (2011). El teletrabajo:c Más libertad o una nueva forma de esclavitud para los trabajadores? IDP. Revista de Internet, Derecho y Política(11).

Porter, M., \& Kramer, M. R. (2011). Creating shared value. Harvard Business Review.

Ruth, S., \& Chaudhry, I. (2008). Telework: A Productivity Paradox? Internet Computing, IEEE, 12(6), 87-90. doi: 10.1109/mic.2008.132

Universia Colombia. (2012). El 71\% de los colombianos es afín al teletrabajo Mercado Laboral. Colombia.

Vari, M., Tagliavini, G., \& Ter-Oganesova, K. (2011, Aug. 31 2011-Sept. 3 2011). Telework: At the crossroads of social demand and technology offer. Paper presented at the FITCE Congress (FITCE), 2011 50th. 